\title{
Single fraction computed tomography-guided high-dose-rate brachytherapy or stereotactic body radiotherapy for primary and metastatic lung tumors?
}

\author{
Mark KH Chan, PhD!', Venus WY Lee, MSc², Noriyuki Kadoya, PhD³, Chi-Leung Chiang, MBChB²,4 Matthew YP Wong, MSc², \\ Ronnie WK Leung, MSc², Steven Cheung, MSc², Oliver Blanck, PhDl \\ 'Department of Radiation Oncology, University Schleswig-Holstein, Kiel Campus, Germany, ²Department of Clinical Oncology, Tuen Mun \\ Hospital, Hong Kong (S.A.R), ${ }^{3}$ Department of Radiation Oncology, Tohoku University Graduate School of Medicine, Japan, ${ }^{4}$ Department of \\ Clinical Oncology, The University of Hong Kong-Shenzhen Hospital, Hong Kong (S.A.R)
}

\begin{abstract}
Purpose: To provide a pilot dosimetric study of computed tomography (CT)-guided high-dose-rate (HDR) brachytherapy (BRT) and stereotactic body radiotherapy (SBRT) for primary and metastatic lung lesions.

Material and methods: For nine lung primary and metastasis patients, 3D image-based BRT plan using a single virtual catheter was planned for $34 \mathrm{~Gy}$ in single fraction to the gross tumor volume (GTV) $+3 \mathrm{~mm}$ margin to account for tumor deformation. These plans were compared to margin-based (MB-) and robustness optimized (RO-) SBRT, assuming the same tumor deformation under real-time tumor tracking. Consistent dose calculation was ensured for both BRT and SBRT plans using the same class of collapsed cone convolution superposition algorithm. Plan quality metrics were compared by Friedman tests and Wilcoxon $t$-tests.

Results and Conclusions: Brachytherapy plans showed significant higher GTV mean dose compared to MB- and RO-SBRT (122.2 Gy vs. 50.4 and $44.7 \mathrm{~Gy}, p<0.05)$, and better dose gradient index $\left(\mathrm{R}_{50}\right)=2.9$ vs. 4.3 and 8.4 for MB- and RO-SBRT, respectively. Dose constraints per the RTOG 0915 protocol were achieved for all critical organs except chest wall in BRT. All other dose-volume histograms (DVH) metrics are comparable between BRT and SBRT. Treatment delivery time of BRT and SBRT plans significantly increased and decreased with increasing GTV size, respectively. SBRT using advanced MLC tracking technique and non-coplanar VMAT can achieve comparable dosimetric quality to HDR BRT. Whether or not, the significantly higher GTV dose can increase killing of radioresistant tumor cells and offset the effect of tumor reoxygenation in single fraction BRT, requires further clinical investigation.
\end{abstract}

J Contemp Brachytherapy 2018; 10, 5: 446-453 DOI: https://doi.org/10.5114/jcb.2018.79335

Key words: CT-guided brachy, HDR, high-dose-rate, lung cancer, metastasis, stereotactic body radiotherapy.

\section{Purpose}

Computed tomography (CT-guided) minimally invasive Iridium-192 high-dose-rate (HDR) brachytherapy (BRT) has been introduced as a form of local radiation treatment for inoperable primary and metastatic lung cancer [1,2]. CT-HDRBRT is performed mostly through collaboration with interventional radiologists for insertion of brachytherapy catheters into the tumor [3]. This internal radioablative technique allows for the localized dose to be delivered in a single session with very rapid dose gradient within a few millimeters around the source to spare the adjacent normal tissues.

In a phase I prospective study, Ricke et al. [4] reported a local tumor control rate (LCR) of $97 \%$ for 15 patients with 30 lung lesions using a minimum dose of $20 \mathrm{~Gy}$.
The follow-up period was, however, short at a median of $5+$ months. The following phase II study included a larger cohort of 30 patients with 83 primary and secondary lung lesions, and they reported at a longer follow up of 12 months LCR of $91 \%$ with the same treatment dose level [5]. In another series comprising 60 lesions from 55 patients, the reported local control rates for metastatic tumors were $93 \%, 82 \%$, and $82 \%$, and for primary intrathoracic cancers were $86 \%, 79 \%$, and $73 \%$ at 1,2 , and 3 years, respectively [6]. A recent phase I trial also investigated combined HDRBRT in single fraction of $30 \mathrm{~Gy}$ with $\geq 70 \mathrm{~Gy}$ intensity modulated radiotherapy (IMRT) in 2 to $2.2 \mathrm{~Gy}$ fractions for regional positive lymph node in locally advanced non-small cell carcinoma, showing 1- and 2-year overall survival rates (OS) of $90.9 \%$ and $67 \%$, and median OS of 22.5 months [7]. Nonetheless, CT-HDRBRT is not

Address for correspondence: Venus WY Lee, MSc, Department of Clinical Oncology, Tuen Mun Hospital, Received: 27.05.2018 Hong Kong (S.A.R), 23 Tsing Chung Koon Road, Tuen Mun, New Territories, Hong Kong, Accepted: 27.09 .2018 phone: +852 2468 5038, e-mail: lwy174@ha.org.hk Published: 30.10 .2018 
frequently used in clinical practice primarily due to necessary intervention, the limited reports of long-term outcomes, the difficulty in gaining expertise with CT-guided interstitial techniques, and the inadequate dosimetry formalism to obtain dose distribution in the low-density heterogeneous lung tissue [8].

The alternative treatment option that has gained widespread popularity is stereotactic body radiotherapy (SBRT). This treatment technique has been associated with mature treatment outcomes demonstrating acceptable toxicities with excellent long-term LCR and OS for both inoperable primary non-small cell lung carcinomas (NSCLC) and secondary metastases $[9,10,11,12]$. However, excessive grade 3 and 4 toxicities including decline pulmonary function tests, pneumonias, skin and pleural reaction were observed for the centrally located lesions from the early experience [13]. With a modified dose schedule of $50 \mathrm{~Gy}$ in 4 fractions, the toxicities of grade 3 and above were successfully reduced to $1 \%$ [14]. Lower normal tissue toxicities in treating these central lesions were also reported using active motion compensation such as robotic or multileaf collimator (MLC) tracking techniques to reduce the dose burden with smaller safety margin [10]. The recently closed Radiation Therapeutic Oncology Group (RTOG) protocol 0915 comparing two dose schedules of $34 \mathrm{~Gy}$ in single fraction and $48 \mathrm{~Gy}$ in 4 fractions further found median OS of 4.1 vs. 4.0 years, and primary tumor failure (PTF) rate of $7.9 \%$ vs. $6.8 \%$ with no excess in late-appearing toxicity observed in either arm [15].

So far, there have been no clinical studies comparing CT-HDRBRT with SBRT for inoperable primary and metastatic lung tumors. In a recent dosimetric comparison, Milickovic et al. [16] showed equivalent target conformity with HDRBRT and sharper dose fall-off than historic SBRT plans based on nine to ten non-coplanar static beams. This study aimed to provide a pilot dosimetric analysis to understand the potential role of HDRBRT and the most state-of-the art SBRT technique realized by inversely optimized dynamic arc delivery through real-time MLC tracking. By using the same category of model-based collapse cone dose calculation engine, this study aimed to compare these two treatment modalities based on accurate estimation of the absorbed dose distributions.

\section{Material and methods}

\section{Patient and treatment prescriptions}

A total of 9 primary or metastatic lung lesions $\leq 5 \mathrm{~cm}$ in diameter were included in this planning study approved by the local ethics committee (NTWC/CREC/15140). Gross tumor volume (GTV) and various organs at risk (OAR) were defined on non-contrast computed tomography at mid-ventilation (MidV-CT). The prescription dose of $34 \mathrm{~Gy}$ in one single fraction chosen in this study was based on the RTOG protocol 0915 [17]. The dose constraint guideline as described in the protocol 0915 was also followed in the planning of CT-HDRBRT and SBRT. The principal planning objective was to achieve the prescription dose to $50 \%$ of the GTV (i.e., median GTV dose
= $34 \mathrm{~Gy}$ ), where the tolerance dose to OAR could not be achieved. For each patient, the same set of planning MidV-CT together with the structure set was exported to the Oncentra ${ }^{\circledR}$ Brachy (OcB; Elekta, Stockholm, Sweden) treatment planning system (TPS) v.4.5 for CT-HDRBRT planning, and to the RayStation TPS v.5.0 (RaySearch Laboratories, Stockholm, Sweden) for SBRT planning. For consistency in dose specification, the same material assignment was used on both TPSs.

\section{Treatment planning for brachytherapy}

High-dose-rate brachytherapy was assumed to be delivered with an ${ }^{192} \mathrm{Ir}$ source of $41152.3 \mu \mathrm{Gy} \mathrm{m} \mathrm{m}^{2} / \mathrm{h}$ from a MicroSelectron ${ }^{\circledR}$ afterloader (Elekta Brachytherapy, Elekta AB, Stockholm, Sweden). For each lesion, a single virtual brachytherapy catheter was used. The route of the virtual needle insertion was defined by an interventional radiologist using the same principle of CT-guided biopsy at our institution. In general, the route was taken to be the shortest possible from where it entered to the tumor's center to minimize the trauma from the procedure. The placement of the brachytherapy catheter was assumed to follow the procedure described by Ricke $e t$ al. [4]. Furthermore, the virtual catheter path was such that it always passed through the tumor's center of mass (COM) and extended beyond the GTV surface by $3 \mathrm{~mm}$ corresponding to a uniform PTV margin. We assumed a coherent rigid movement of the brachytherapy catheter together with the tumor in presence of respiratory motion. Hence, the safety margin was needed to compensate only for the tumor deformation, which was measured from four-dimensional computed tomography (4D-CT) to be $2.7 \mathrm{~mm}$ at maximum in the craniocaudal direction [18].

Where necessary and technically feasible, the long axis of the source was oriented such that the dose to adjacent OAR was optimized by taking advantage of the anisotropic dose distribution. The source was manually loaded to one or multiple dwell positions, depending on the tumor size and the proximity of the tumor to the OAR. For small lesions, a single dwell position at the GTV's COM was generally sufficient, while for large lesion more than one source dwell positions were needed to yield better dosimetry. For all plans, dwell weights of the source were optimized in a manual manner.

Oncentra TPS offers two options for dose calculation: the TG-43 formalism and the model-based Advanced Collapsed cone Engine (ACE). The TG-43 formalism is the most clinically used method in brachytherapy treatment planning, but it is well known for its limitation of calculating absorbed dose to a semi-infinite water phantom only. It neglects the reduced attenuation and different scattering property of the lung tissue relative to water in the finite patient geometry. Hence, this study abandoned the TG-43 and used ACE in all dose calculations.

ACE models separated the dose depositions by primary and scatter photons by scaling the discretized point kernels according to the material type and density along each radiation transport line (i.e., the conical axis in the implementation of the collapsed cone algorithm). To enable the heterogeneity corrections with ACE, each contour 
associated with the patient CT was assigned with a material of variable density according to the CT Hounsfield unit (HU). For this study, the lung contour was assigned as inflated lung whose elemental compositions were defined as in the TG 186 report [8]. For other hollow organs, which included the trachea, bronchi, and esophagus, the contours were segmented into air cavities and surrounding walls with assigned material types of air and water, respectively. The GTV and all other contours were also assigned as water. This yielded an absorbed dose distribution to media $\mathrm{D}_{\mathrm{m}, \mathrm{m}}$.

\section{Treatment planning of SBRT}

Treatment plans were developed for a demonstration-purposed TrueBeam linac (Varian Inc., Palo Alto, CA, USA) to deliver volumetric-modulated arc radiotherapy (VMAT)-based SBRT in HDR flattening filter-free (FFF) mode. Two to three partial coplanar and non-coplanar arcs using either $10 \mathrm{MV}$ or mixed $6 \mathrm{MV}$ and $10 \mathrm{MV}$ photon beams were used aiming to achieve the best possible sparing of the normal tissues $[19,20]$. The uniform $3 \mathrm{~mm}$ GTV-to-PTV margin for tumor deformation was the same as in CT-HDRBRT, assuming perfect image-guided realtime tumor tracking by multileaf collimator (MLC) as active motion management [21]. It is worthwhile noting that VMAT-based MLC-tracking SBRT in FFF mode has not been yet implemented clinically. Nonetheless, VMATbased SBRT delivery in FFF mode was reported in a few clinical studies showing 1-year local control of $94 \%$ for primary and metastatic lung lesions [22], and 100\% in early stage NSCLC [23]. The clinical implementation of real-time adaptive electromagnetic transponder-guided MLC tracking was also reported in SBRT of lung by Booth et al. [24], demonstrating great potential of this motion compensation technique to ensure the delivered dose to $95 \%\left(D_{95}\right)$ of GTV of $110 \pm 0.5 \%$ compared to $110 \%$ planned. A clinical trial for lung cancers using MLC tracking has been also registered by the same group recently [24].

This study compared two VMAT planning approaches with CT-HDRBRT, either using the conventional concept of PTV or based on the robust optimization method to explicitly accounts for the geometric tracking error, showing $3 \mathrm{D}$ root-mean square of $0.5 \mathrm{~mm}$ [25] and the tumor deformation of $2.7 \mathrm{~mm}$. The total geometric error was rounded to $3 \mathrm{~mm}$ in this study. The resulting SBRT plans were abbreviated as MB-SBRT (margin-based SBRT) and ROSBRT (robust optimized), respectively. The RayStation TPS achieves robustness by minimax optimization, in which optimization functions are considered in a worst-case scenario. The set of scenarios forms a discretization of the userspecified errors against, which the plan is robust from both the GTV' and the OARs' perspectives. Robustness was imposed to all dose-volume constraints of the serial organs and the GTV. This study further exploited the lateral electronic disequilibrium phenomenon to maximize the dose gradient at the lung-tumor interface [26], increasing the dose by as much as $40 \%$ (i.e., $60 \%$ isodose level relative to the maximum dose) to the PTV or GTV, depending on the optimization scenarios, to emulate brachytherapy. Higher PTV maximum dose was also reported to be asso- ciated with improved local control [27]. For consistency in the dose reporting, the same tissue materials as defined for brachytherapy were used in all SBRT plans. The final dose distribution was calculated by a collapse-cone convolution superposition dose engine with a $2.0 \mathrm{~mm}$ grid. The dose specification was by default $\mathrm{D}_{\mathrm{m}, \mathrm{m}}$.

For MB-SBRT, the prescription dose was normalized to the same level as for HDRBRT per case, which demanded that at least $95 \%$ of the PTV and $99 \%$ of the GTV received 34 Gy. For RO-SBRT, the dose normalization was based on the at least 99\% GTV receiving $34 \mathrm{~Gy}$.

\section{Statistical comparisons of dosimetric metrics}

HDRBRT and SBRT were compared following the dose constraints in the RTOG0915 protocol. Additionally, the absolute normal lung (bilateral lung minus GTV) and the ipsilateral lung volumes receiving at least $20 \mathrm{~Gy}$, $10 \mathrm{~Gy}$, and $5 \mathrm{~Gy}$ (i.e., $\mathrm{V}_{20}, \mathrm{~V}_{10}$, and $\mathrm{V}_{5}$ ) were compared. The plan quality was also quantified using the dose conformity index $(\mathrm{CI})$ defined as

$$
\mathrm{CI}=\mathrm{TV}_{\mathrm{PIV}} \times \mathrm{TV}_{\mathrm{PIV}} /(\mathrm{TV} \times \mathrm{PIV})
$$

where $\mathrm{TV}_{\mathrm{PIV}}, \mathrm{TV}$, and PIV are the volume of target covered by prescription dose, the target volume, and prescription dose volume, respectively [28]. The volume ratio of 17 Gy to $34 \mathrm{~Gy}\left(\mathrm{R}_{50}\right)$ was used to assess the dose gradient achieved with the two treatment approaches. Comparisons of treatment length were based on the estimates from the Oncentra Brachy for HDRBRT and RayStation for SBRT, respectively.

For statistical comparisons of the above dose metrics and those in the RTOG 0915 protocol, Friedman tests were performed with further post hoc Wilcoxon $t$-tests comparison if significance were reached. For correlation of estimated treatment time with the PTV size, the Spearman correlations were used. All statistical tests were run using the Statistical toolbox in Matlab ${ }^{\circledR}$ R2014a (MathWork, Natick, MA, USA).

\section{Results}

Table 1 summarizes the dosimetric and plan quality results over all patients. The GTV ranged from $0.6-20.3 \mathrm{~cm}^{3}$, with a mean of $7.2 \mathrm{~cm}^{3}$.

\section{Organ at risks dose statistics}

Dose constraints per the RTOG 0915 protocol were achieved for all OARs, except for the chest wall/ ribs. HDRBRT exceeded the volume maximum dose of $22 \mathrm{~Gy}$ limited to $1 \mathrm{~cm}^{3}\left(\mathrm{~V}_{22 \mathrm{~Gy}}\right)$ by 1.17 and $0.62 \mathrm{~cm}^{3}$ in two plans, and maximum point dose of $30 \mathrm{~Gy}$ by $19.8 \mathrm{~Gy}$ in one plan. The ribs volume of $1 \mathrm{~cm}^{3}$ limited to $22 \mathrm{~Gy}\left(\mathrm{~V}_{22 \mathrm{~Gy}}\right)$ was exceeded in two HDRBRT plans by 1.17 and 0.62 $\mathrm{cm}^{3}$, in two MB-SBRT plans by 1.5 and $1.4 \mathrm{~cm}^{3}$, and in one RO-SBRT plan by $0.4 \mathrm{~cm}^{3}$, respectively. The maximum ribs dose $\mathrm{D}_{\max }$ was exceeded in the same patient by $19.8 \mathrm{~Gy}$ for HDRBRT, 10.5 Gy for MB-SBRT, and 8.8 Gy for RO-SBRT, respectively. The overall means of ribs $V_{22 G y}$ and $D_{\max }$ were within the RTOG constraints. In Figure 1 , 
Table 1. Dosimetric results from different treatment modalities

\begin{tabular}{|c|c|c|c|}
\hline & HDRBRT & MB-SBRT & RO-SBRT \\
\hline \multicolumn{4}{|l|}{ Normal total lung } \\
\hline $\mathrm{D}_{1500 \mathrm{~cm}^{3}}(\mathrm{~Gy})$ & $0.6 \pm 0.6$ & $0.2 \pm 0.2$ & $0.2 \pm 0.2$ \\
\hline $\mathrm{D}_{1000 \mathrm{~cm}^{3}}(\mathrm{~Gy})$ & $0.9 \pm 0.9$ & $0.2 \pm 0.5$ & $0.2 \pm 0.3$ \\
\hline $\mathrm{V}_{5 \mathrm{~Gy}}\left(\mathrm{~cm}^{3}\right)$ & $239.0 \pm 136.9$ & $237.7 \pm 92.7$ & $230.2 \pm 115.0$ \\
\hline $\mathrm{V}_{7 \mathrm{~Gy}}\left(\mathrm{~cm}^{3}\right)$ & $153.0 \pm 90.2$ & $156.0 \pm 84.9$ & $168.9 \pm 92.0$ \\
\hline $\mathrm{V}_{10 G y}\left(\mathrm{~cm}^{3}\right)$ & $97.3 \pm 59.9$ & $109.4 \pm 51.9$ & $114.5 \pm 68.5$ \\
\hline $\mathrm{V}_{20 \mathrm{~Gy}}\left(\mathrm{~cm}^{3}\right)$ & $34.7 \pm 21.3$ & $36.0 \pm 19.9$ & $37.7 \pm 27.1$ \\
\hline $\mathrm{V}_{7.4 \mathrm{~Gy}}\left(\mathrm{~cm}^{3}\right)$ & $145.0 \pm 87.0$ & $157.3 \pm 65.6$ & $160.0 \pm 88.5$ \\
\hline \multicolumn{4}{|c|}{ Ipsilateral normal lung } \\
\hline $\mathrm{V}_{5 \mathrm{~Gy}}\left(\mathrm{~cm}^{3}\right)$ & $234.8 \pm 131.0$ & $229.1 \pm 86.0$ & $224.1 \pm 106.4$ \\
\hline $\mathrm{V}_{10 G y}\left(\mathrm{~cm}^{3}\right)$ & $91.1 \pm 60.0$ & $106.2 \pm 47.6$ & $110.9 \pm 63.7$ \\
\hline $\mathrm{V}_{\text {20Gy }}\left(\mathrm{cm}^{3}\right)$ & $32.5 \pm 20.2$ & $34.2 \pm 17.1$ & $35.9 \pm 25.0$ \\
\hline \multicolumn{4}{|l|}{ Trachea } \\
\hline$\overline{D_{4 \mathrm{~cm}^{3}}(\mathrm{~Gy})}$ & $1.6 \pm 2.1$ & $1.9 \pm 3.4$ & $1.3 \pm 2.6$ \\
\hline$\overline{V_{10.5 G y}\left(\mathrm{~cm}^{3}\right)}$ & $0.0 \pm 0.0$ & $0.3 \pm 0.9$ & $0.0 \pm 0.0$ \\
\hline $\mathrm{D}_{\max }(\mathrm{Gy})$ & $4.7 \pm 2.7$ & $4.4 \pm 5.5$ & $3.3 \pm 4.3$ \\
\hline \multicolumn{4}{|l|}{ Bronchus } \\
\hline $\mathrm{D}_{4 \mathrm{~cm}^{3}}(\mathrm{~Gy})$ & $2.1 \pm 2.2$ & $1.9 \pm 2.0$ & $1.7 \pm 2.0$ \\
\hline$\overline{V_{10.5 G y}}\left(\mathrm{~cm}^{3}\right)$ & $0.1 \pm 0.1$ & $0.2 \pm 0.3$ & $0.0 \pm 0.1$ \\
\hline $\mathrm{D}_{\max }(\mathrm{Gy})$ & $7.8 \pm 4.5$ & $9.9 \pm 6.3$ & $8.0 \pm 6.1$ \\
\hline \multicolumn{4}{|l|}{ Vessels } \\
\hline $\mathrm{D}_{10 \mathrm{~cm}^{3}}(\mathrm{~Gy})$ & $3.2 \pm 5.5$ & $3.9 \pm 4.8$ & $2.9 \pm 4.3$ \\
\hline $\mathrm{V}_{31 \mathrm{~Gy}}\left(\mathrm{~cm}^{3}\right)$ & $0.1 \pm 0.3$ & $0.0 \pm 0.0$ & $0.1 \pm 0.2$ \\
\hline$\overline{D_{\max }}(G y)$ & $14.2 \pm 15.9$ & $16.5 \pm 15.4$ & $15.0 \pm 13.9$ \\
\hline \multicolumn{4}{|l|}{ Heart } \\
\hline $\mathrm{V}_{16 \mathrm{~Gy}}\left(\mathrm{~cm}^{3}\right)$ & $0.4 \pm 1.2$ & $0.3 \pm 0.9$ & $0.3 \pm 0.5$ \\
\hline $\mathrm{D}_{15 \mathrm{~cm}^{3}}(\mathrm{~Gy})$ & $3.4 \pm 3.2$ & $3.9 \pm 3.8$ & $3.6 \pm 4.1$ \\
\hline $\mathrm{D}_{\max }(\mathrm{Gy})$ & $9.4 \pm 8.1$ & $9.2 \pm 8.1$ & $10.2 \pm 9.1$ \\
\hline \multicolumn{4}{|l|}{ Esophagus } \\
\hline $\mathrm{V}_{11.9 \mathrm{~Gy}}\left(\mathrm{~cm}^{3}\right)$ & $0.0 \pm 0.1$ & $0.0 \pm 0.0$ & $0.0 \pm 0.0$ \\
\hline $\mathrm{D}_{5 \mathrm{~cm}^{3}}(\mathrm{~Gy})$ & $0.9 \pm 1.3$ & $1.4 \pm 2.5$ & $1.1 \pm 1.9$ \\
\hline $\mathrm{D}_{\max }(\mathrm{Gy})$ & $3.7 \pm 2.0$ & $4.9 \pm 2.8$ & $3.6 \pm 2.2$ \\
\hline \multicolumn{4}{|l|}{ Chest wall and ribs } \\
\hline $\mathrm{D}_{1 \mathrm{~cm}^{3}}(\mathrm{~Gy})$ & $15.2 \pm 7.8$ & $18.4 \pm 5.4$ & $17.5 \pm 4.8$ \\
\hline$\overline{V_{31 G y}\left(\mathrm{~cm}^{3}\right)}$ & $0.1 \pm 0.2$ & $0.0 \pm 0.1$ & $0.0 \pm 0.0$ \\
\hline $\mathrm{D}_{\max }(\mathrm{Gy})$ & $22.6 \pm 12.8$ & $25.0 \pm 8.9$ & $22.9 \pm 8.5$ \\
\hline $\mathrm{V}_{\text {22Gy }}\left(\mathrm{cm}^{3}\right)$ & $0.5 \pm 0.8$ & $0.7 \pm 1.0$ & $0.3 \pm 0.5$ \\
\hline \multicolumn{4}{|l|}{ PTV } \\
\hline$\overline{V_{34 G y}(\%)}$ & $96.2 \pm 0.7$ & $96.2 \pm 0.7$ & N/A \\
\hline $\mathrm{V}_{30.6 \mathrm{~Gy}}(\%)$ & $99.0 \pm 0.0$ & $99.5 \pm 0.3$ & $\mathrm{~N} / \mathrm{A}$ \\
\hline $\mathrm{D}_{\text {mean }}(\mathrm{Gy})$ & $65.0 \pm 5.2$ & $43.3 \pm 1.7$ & $\mathrm{~N} / \mathrm{A}$ \\
\hline \multicolumn{4}{|l|}{ GTV } \\
\hline $\mathrm{V}_{34 \mathrm{~Gy}}(\%)$ & $100.0 \pm 0.0$ & $100.0 \pm 0.0$ & $99.0 \pm 0.1$ \\
\hline $\mathrm{D}_{\text {median }}(\mathrm{Gy})$ & $122.2 \pm 19.0$ & $50.4 \pm 1.5$ & $44.7 \pm 3.9$ \\
\hline \multicolumn{4}{|l|}{ Spinal cord } \\
\hline $\mathrm{D}_{1.2 \mathrm{~cm}^{3}}(\mathrm{~Gy})$ & $1.3 \pm 1.1$ & $2.9 \pm 2.1$ & $2.1 \pm 1.2$ \\
\hline $\mathrm{D}_{0.35 \mathrm{~cm}^{3}}(\mathrm{~Gy})$ & $1.7 \pm 1.4$ & $3.4 \pm 2.4$ & $2.4 \pm 1.4$ \\
\hline$\overline{D_{\max }}(G y)$ & $3.1 \pm 2.0$ & $4.0 \pm 2.6$ & $3.0 \pm 1.8$ \\
\hline Conformity index & $0.7 \pm 0.1$ & $0.9 \pm 0.0$ & $0.7 \pm 0.1$ \\
\hline$R_{50}$ & $2.9 \pm 0.0$ & $4.3 \pm 1.4$ & $8.4 \pm 4.1$ \\
\hline
\end{tabular}

PTV - planning target volume; GTV - gross tumor volume; HDRBRT - high-dose-rate brachytherapy plan; MB-SBRT - margin-base stereotactic radiotherapy plan;

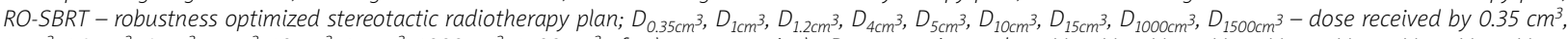

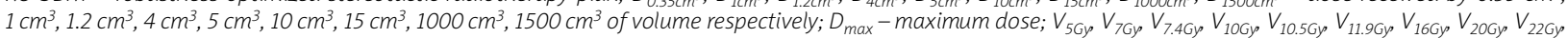
$V_{30,6 G,} V_{31 G y}$ and $V_{34 G y}$ - volume received by $5 \mathrm{~Gy}, 7 \mathrm{~Gy}, 7.4 \mathrm{~Gy}, 10 \mathrm{~Gy}, 10.5 \mathrm{~Gy}, 11.9 \mathrm{~Gy}, 16 \mathrm{~Gy}, 20 \mathrm{~Gy}, 22 \mathrm{~Gy}, 30.6 \mathrm{~Gy}, 31 \mathrm{~Gy}$, and $34 \mathrm{~Gy}$ respectively; $R_{50}$ - gradient index, which defined as a ratio of the $50 \%$ prescription isodose line to PTV volume 


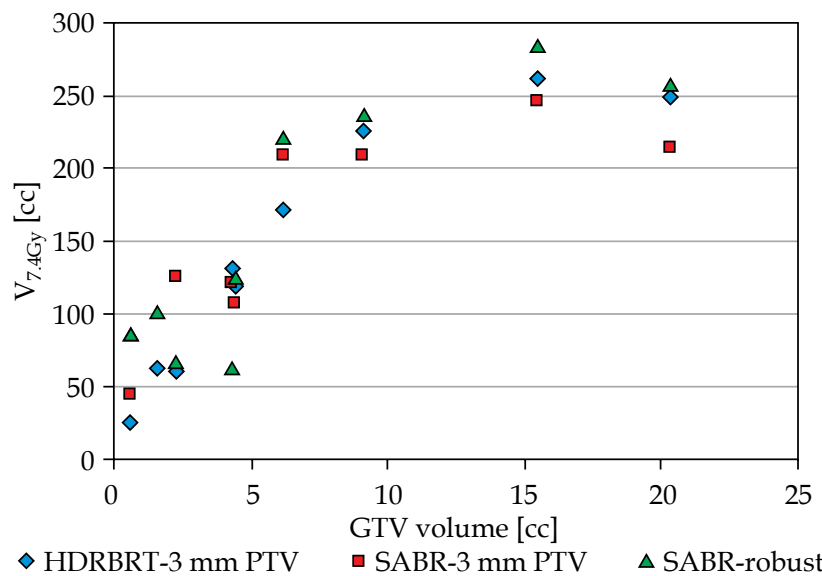

Fig. 1. Total lung volume receiving at least 7.4 Gy of individual lesions in order of increasing size

the normal total lung volume receiving no more than $7.4 \mathrm{~Gy}$, which is associated with the risk of pneumonitis, is shown. The low (5 Gy) to high (20 Gy) dose volumes to the ipsilateral lung are also included in Figure 1. Statistically, all studied DVH metrics related to normal tissue showed no differences among treatment methods. Figure 2 shows the dose distributions obtained with HDRBRT, MB-, and RO-SBRT for an anterior and the other peripheral lesions.

\section{GTV and PTV dose statistics}

The prescription dose coverage $\left(\mathrm{V}_{34 \mathrm{~Gy}}\right)$ to the PTV was normalized to $\geq 95 \%$, and $V_{34 G y}$ for GTV $=100 \%$ for all lesions in HDRBRT and MB-SBRT (Table 1). Figure 3 presents the GTV median dose for individual patients.
The averaged GTV mean dose is $122.19 \pm 19.0$ Gy (one standard deviation: SD) for HDRBRT, $50.4 \pm 1.5$ Gy for MB-SBRT, and 44.7 \pm 3.9 Gy for RO-SBRT. Pair-wise comparisons showed significant higher GTV mean dose with HDRBRT than MB- and RO-SBRT $(p<0.01)$. Mean doses to the PTV for HDRBRT and MB-SBRT were 65.1 $\pm 5.2 \mathrm{~Gy}$ and $43.4 \pm 1.7 \mathrm{~Gy}$, respectively $(p<0.01)$. Likewise, mean doses to the PTV border zone (i.e., PTV minus GTV) were also significantly higher $(p<0.01)$ for HDRBRT (51.5 $\pm 3.8 \mathrm{~Gy})$ than for MB-SBRT (39.6 $\pm 0.8 \mathrm{~Gy})$.

\section{Plan quality index and treatment delivery efficiency}

Other plan quality metrics of $\mathrm{CI}$ and the dose gradient index $\left(R_{50}\right)$ are summarized in Figure 4. The mean CI for the HDRBRT plans was 0.7 (range: 0.6-0.8), for MB-SBRT plans 0.9 (range: 0.8-0.9), and for the RO-SBRT plans 0.7 (range: $0.4-0.8$ ). Statistical tests showed significantly better CI with MB-SBRT than HDRBRT and RO-SBRT $(p<0.01)$. The dose fall gradient in terms of $\mathbf{R}_{50}$ showed means of $2.9 \pm 0.0$ for HDRBRT, $4.3 \pm 1.4$ for MB-SBRT, and $8.4 \pm 4.1$ RO-SBRT, respectively. They were statistically different between HDRBRT and MB- $(p<0.05)$ and RO-SBRT $(p<0.01)$.

The averaged treatment lengths varied from $12.3 \mathrm{~min}-$ utes (range: 3.5-24.3 minutes) for HDRBRT, 5.4 minutes (range: 2.8-10.4 minutes) for RO-SBRT, to 4.7 minutes (range: 3.2-7.0 minutes) for MB-SBRT.

\section{Correlation of plan quality parameters with GTV size}

Table 2 presents the results of correlations between the GTV size and selected plan evaluation metrics. HDRBRT
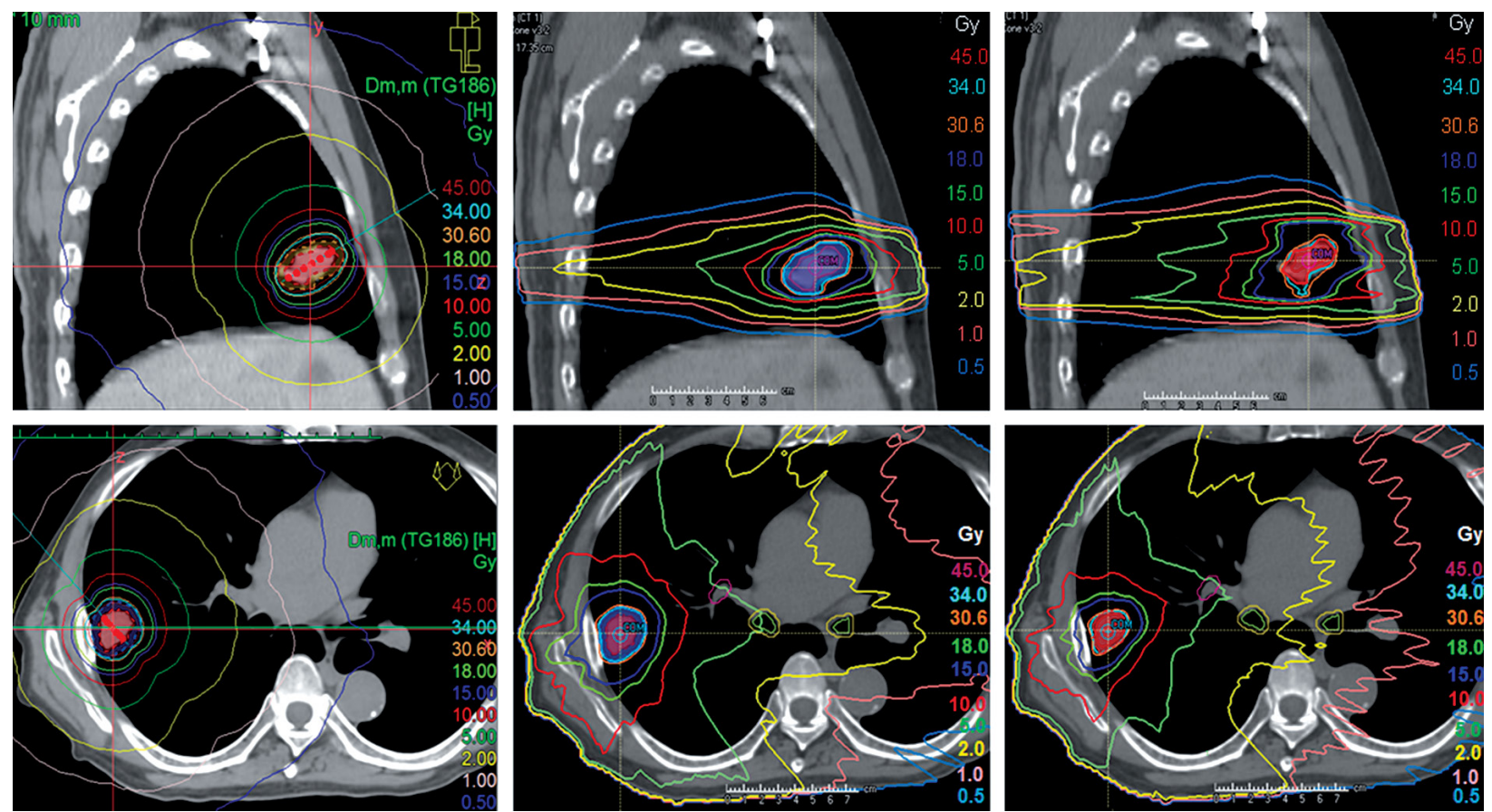

Fig. 2. Top row (from left to right) shows dose distributions of HDRBRT, MB-SBRT, and RO-SBRT for an anterior lesion, and bottom row for a peripheral lesion. For the anterior lesion, the needle path was angled to align with the long axis of the lesion in HDRBRT 
shows a trend of increasing GTV mean dose with decreasing GTV size. Similar trend was observed for RO-SBRT but not as obvious as for MB-SBRT. Correlations between treatment delivery time and GTV size were significant and were reciprocal for HDRBRT and MB-/RO-SBRT.

\section{Discussion}

Brachytherapy offers the potential of highly localized dose deposition with very sharp fall-off. This dosimetric characteristic makes it the ideal option as monotherapy and/or additive therapy for prostate and cervix cancers. However, its role in inoperable early stage NSCLC is not clearly defined as SBRT remains the preferred option due to its evidenced safety and efficacy through large scale clinical trials $[9,15]$.

Unlike previous in silico study comparing HDRBRT to relatively obsolete SBRT techniques with unclear definition of safety margin [16], this study assessed the dosimetric quality in a more objective setting by assuming the most advanced technologies available to both SBRT and HDRBRT. Whilst real-time MLC tracking is far from a standard technique in clinical routine of SBRT and is exclusively trialed for lung SBRT [24], its realization made it possible to reduce or completely remove the safety margin by robustness augmentation [21]. The resulting lung doses to the ipsilateral lung in the low to high range were found to have negligible difference from those of HDRBRT. Interestingly, at low-dose of $5 \mathrm{~Gy}$, the lung volume showed highest $\mathrm{V}_{5 \mathrm{~Gy}}$ for HDRBRT, differing from MB-SBRT by 1.3 and $5.7 \mathrm{~cm}^{3}$, and RO-SBRT by 8.8 and $10.7 \mathrm{~cm}^{3}$ on average for the bilateral and ipsilateral lungs, respectively. From $V_{7 G y}$ onward to $V_{20 G y}$, the trend was reversed with HDR BRT showing the lowest averages, which differed from the highest for RO-SBRT by 17.2 and $19.8 \mathrm{~cm}^{3}$ (10 Gy), and 3.0 and $3.4 \mathrm{~cm}^{3}$ (20 Gy) in bilateral and ipsilateral lungs. The significance of reduced contralateral lung dose can be seen in Figure 2. By contrast, SBRT was distinctively associated with smaller low-dose volume in the axial direction even by non-coplanar delivery, as demonstrated for the anterior lesion in Figure 2. From Table 2, significant and positive correlations between the GTV size and the lung dose volume metrics from $V_{5 G y}$ to $V_{20 G y}$ were observed in all treatment modali-

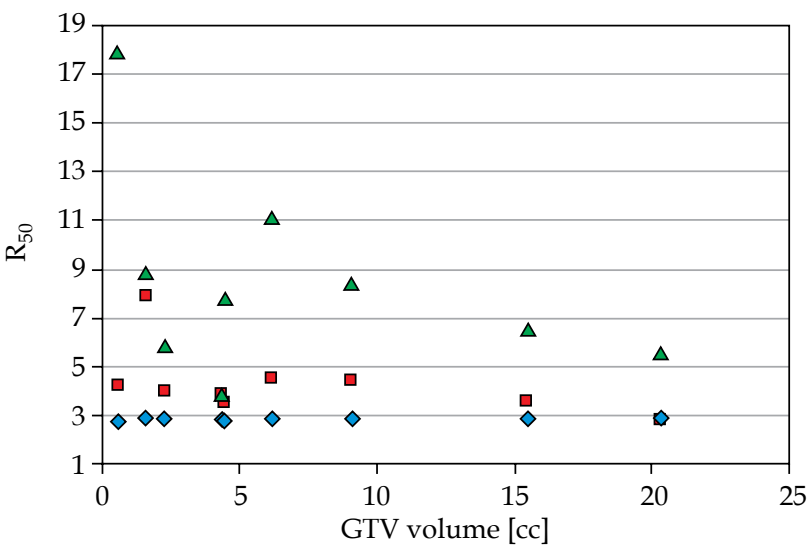

$\diamond$ HDRBRT-3 mm PTV

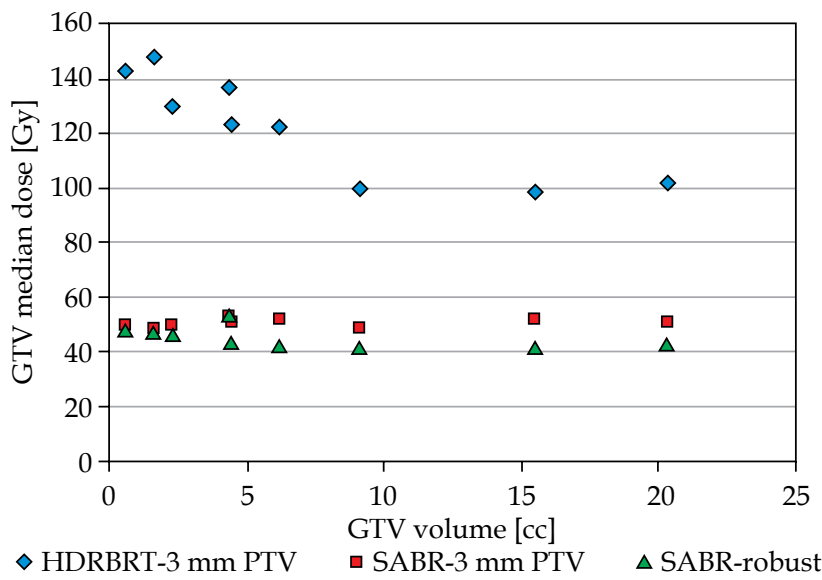

Fig. 3. GTV median dose as a function of GTV size

ties. These results were the combined consequences of the shorter range of the ${ }^{192} \mathrm{Ir} \gamma$ rays (average photon energy of $397 \mathrm{keV}$ vs. $6-10 \mathrm{MeV}$ in SBRT) and internal irradiation, producing faster dose fall-off, and hence much better $\mathrm{R}_{50}$ for HDRBRT than MB- and RO-SBRT.

HDRBRT and SBRT showed reciprocal correlations between treatment length and GTV size. The treatment length increased for HDRBRT with increasing tumor size because more dwell positions and dwell times per unit source strength is required for the prescribed dose, and for SBRT, it decreased with increasing tumor size and hence, increasing output factor. Note that the length for the HDRBRT treatment was calculated based on the assumption of a source strength of $41152.3 \mu \mathrm{Gym}^{2} / \mathrm{h}$. As the ${ }^{192}$ Ir source decays, the treatment length would be even more. According to the linear fits, HDRBRT would take longer treatment durations than MB- and RO-SBRT, when the GTV is larger than 1.5 and $0.2 \mathrm{~cm}^{3}$ with corresponding diameter of 14 and $8 \mathrm{~mm}$, respectively.

With the limitation of minimized needle insertion path through the lung tissue, the source path may not always be in the optimal orientation. Together with the limitation of single catheter versus multiple catheters, may not often produce very conformal dose distribution even by optimized source dwell position and time (Figure 4). The dose conformity index (CI) was HDRBRT, therefore worse for HDRBRT than MB-SBRT but better than

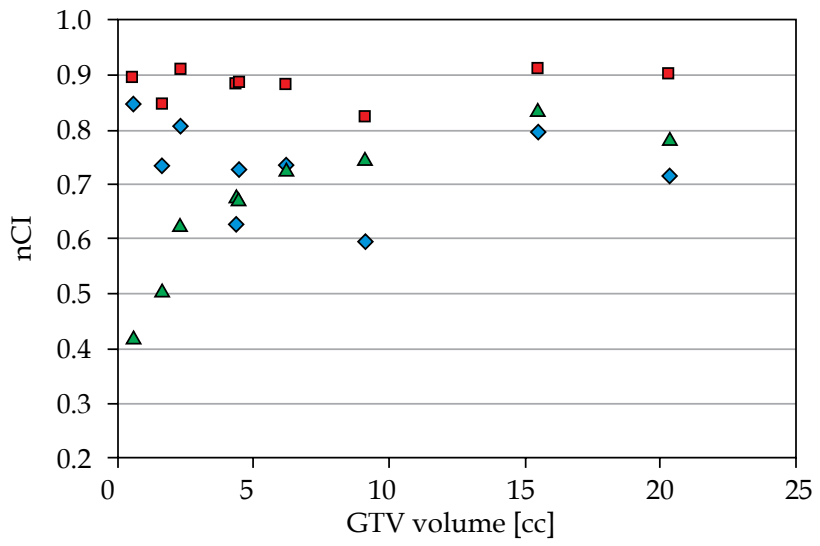

Fig. 4. Dose gradient index $\mathrm{R}_{50}$ and dose conformity index $\mathrm{CI}$ of individual lesions 
Table 2. Correlation between size of gross tumor volume and plan quality metrics

\begin{tabular}{|c|c|c|c|c|c|c|}
\hline & \multicolumn{2}{|c|}{ HDRBRT } & \multicolumn{2}{|c|}{ MB-SBRT } & \multicolumn{2}{|c|}{ RO-SBRT } \\
\hline & $r_{0}$ & $p$-value & $r_{0}$ & $p$-value & $r_{0}$ & $p$-value \\
\hline \multicolumn{7}{|l|}{ Normal total lung } \\
\hline$\underline{\mathrm{D}_{1500 \mathrm{~cm}^{3}}}(\mathrm{~Gy})$ & 0.69 & 0.04 & 0.10 & 0.80 & 0.06 & 0.88 \\
\hline $\mathrm{D}_{1000 \mathrm{~cm}^{3}}(\mathrm{~Gy})$ & 0.71 & 0.03 & -0.08 & 0.83 & 0.63 & 0.07 \\
\hline$\underline{V_{5 G y}}\left(\mathrm{~cm}^{3}\right)$ & 0.97 & 0.00 & 0.85 & 0.00 & 0.92 & 0.00 \\
\hline $\mathrm{V}_{10 \mathrm{~Gy}}\left(\mathrm{~cm}^{3}\right)$ & 0.95 & 0.00 & 0.80 & 0.01 & 0.83 & 0.01 \\
\hline$V_{20 G y}\left(\mathrm{~cm}^{3}\right)$ & 0.98 & 0.00 & 0.88 & 0.00 & 0.85 & 0.00 \\
\hline$V_{7.4 G y}\left(\mathrm{~cm}^{3}\right)$ & 0.95 & 0.00 & 0.82 & 0.01 & 0.83 & 0.01 \\
\hline \multicolumn{7}{|l|}{ Ipsilateral normal lung } \\
\hline $\mathrm{V}_{5 \mathrm{~Gy}}\left(\mathrm{~cm}^{3}\right)$ & 0.98 & 0.00 & 0.85 & 0.00 & 0.93 & 0.00 \\
\hline $\mathrm{V}_{10 \mathrm{~Gy}}\left(\mathrm{~cm}^{3}\right)$ & 0.93 & 0.00 & 0.78 & 0.01 & 0.83 & 0.01 \\
\hline $\mathrm{V}_{20 \mathrm{~Gy}}\left(\mathrm{~cm}^{3}\right)$ & 0.95 & 0.00 & 0.90 & 0.00 & 0.83 & 0.01 \\
\hline \multicolumn{7}{|l|}{ GTV } \\
\hline $\mathrm{D}_{\text {mean }}(\mathrm{Gy})$ & -0.92 & 0.01 & 0.27 & 0.49 & -0.80 & 0.01 \\
\hline \multicolumn{7}{|l|}{ PTV-GTV ring } \\
\hline $\mathrm{D}_{\text {mean }}(\mathrm{Gy})$ & -0.42 & 0.27 & -0.18 & 0.64 & & \\
\hline Conformity index & 0.50 & 0.17 & -0.03 & 0.93 & -0.97 & 0.00 \\
\hline Delivery time (seconds) & 0.93 & 0.00 & -0.78 & 0.01 & -0.72 & 0.03 \\
\hline
\end{tabular}

GTV-gross tumor volume; PTV - planning target volume; HDRBRT - high-dose-rate brachytherapy plan; MB-SBRT-the margin-base stereotactic radiotherapy plan;

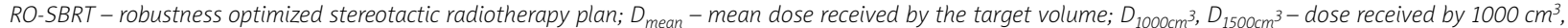
$1500 \mathrm{~cm}^{3}$ of volume, respectively; $V_{5 G y}, V_{7.4 G y} V_{10 G y}, V_{20 G y}$ - volume received by $5 \mathrm{~Gy}, 7.4 \mathrm{~Gy}, 10 \mathrm{~Gy}, 20 \mathrm{~Gy}$, respectively

RO-SBRT. These results disagreed with the previous study, where insignificant but better CI was achieved with HDRBRT than SBRT. These contradicting results were likely because the difference in number of catheters used (6-10 in Milickovic et al. [16] vs. one in the present study). Yet, the average CI in the present study was still better than that achieved by Yang et al. [1] with ideal needle configuration in terms of number of needles and placement ( 0.6 vs. 0.4$)$, indicating that inter-center variability can be significant and limits the generalization of results from different dosimetric studies.

Due to the same limitations, doses to organs at risk, ribs, and chest walls in particular, may in some cases not be well controlled in order to achieve the primary objective of prescription dose coverage for the PTV and GTV. Although the RTOG 0915 protocol does allow some leeway to chest wall dose constraints, maximum dose to the ribs as high as $49.8 \mathrm{~Gy}$ in single fraction has never been reported, and its clinical implication on ribs fracture and chest wall pain remains a genuine concern. In contrast, the large degree of freedom offered by non-coplanar VMAT enables better dose control in certain preferential directions. This directly resulted in lower maximum ribs/ chest wall dose (despite very limited arc range to minimize the radiation path through the healthy lung tissues). Doses to the other critical organs were found to be minimal for either HDRBRT or SBRT because the tumors were generally located at a distance by selection.

Although CT-guided percutaneous single catheter insertion is a minimally invasive procedure, pneumothorax and hemothorax remains a serious concern. The uncertainty of needle and needle displacement due to motion is largely unknown despite promising outcomes. On the oth- er hand, almost all real time tumor tracking technologies today require the implantation of 3 to 4 fiducial markers to allow six degrees of freedom for motion correction [11,24]. The incident rate of pneumothorax associated with fiducial implantation was reported in the range of $26.7 \%$ to $33 \%$ $[29,30]$, which was slightly higher than the $11.5 \%$ to $23.3 \%$ for single or multiple needle insertion in HDRBRT [4,7]. For this possible reason, most clinical studies reported HDRBRT by once or twice daily fractions on a single day. Such limitation may negate the tumor cell killing by tumor re-oxygenation and SBRT treatment should be fractionated [31,32]. Nonetheless, the GTV median dose in HDRBRT was over 70 Gy higher than that in SBRT $(p<0.01)$. Whether this significant higher dose is needed to increase killing of the radioresistant tumors and potentially offset the effect of the tumor re-oxygenation, remains the focal point of clinical investigation, as it will offer important insight into the results of RTOG 0915 trial comparing two arms of single versus multiple fractions.

\section{Acknowledgement}

The authors are grateful for the help from Dr. K.L. Siu of the Department of Interventional Radiology of Tuen Mun Hospital on identifying the patient candidates and performing the virtual needle placement in the HDRBRT simulations. This study was partially supported by a research grant from the Japanese Society for MicroSelectron HDR.

\section{Disclosure}

Authors report no conflict of interest. 


\section{References}

1. Yang B, Sun X, Pang H et al. Dosimetric analysis of rib interference of the CTV during interstitial brachytherapy of lung tumors. J Contemp Brachytherapy 2017; 9: 566-571.

2. Sharma M, Tandon $S$, Nayak U et al. Computerized tomography-guided percutaneous high-dose-rate interstitial brachytherapy for malignant lung lesions. J Cancer Res Ther 2011; 7: 174-179.

3. Bretschneider T, Ricke J, Gebauer B, Streitparth F. Image-guided high-dose-rate brachytherapy of malignancies in various inner organs - technique, indications, and perspectives. J Contemp Brachytherapy 2016; 8: 251-261.

4. Ricke J, Wust P, Wieners G et al. CT-guided interstitial single-fraction brachytherapy of lung tumors: phase I results of a novel technique. Chest 2005; 127: 2237-2242.

5. Peters N, Wieners G, Pech M et al. CT-guided interstitial brachytherapy of primary and secondary lung malignancies. Strahlenther Onkol 2008; 184: 296-301.

6. Tselis N, Ferentinos K, Kolotas C et al. Computed tomography-guided interstitial high-dose-rate brachytherapy in the local treatment of primary and secondary intrathoracic malignancies. J Thorac Oncol 2011; 6: 545-552.

7. Xiang L, Zhang JW, Lin S et al. Computed tomography-guided interstitial high-dose-rate brachytherapy in combination with regional positive lymph node intensity-modulated radiation therapy in locally advanced peripheral non-small cell lung cancer: a phase 1 clinical trial. Int J Radiat Oncol Biol Phys 2015; 92: 1027-1034

8. Beaulieu L, Carlsson $\AA$, Tedgren, Carrier JF et al. Report of the Task Group 186 on model-based dose calculation methods in brachytherapy beyond the TG-43 formalism: Current status and recommendations for clinical implementation. Med Phys 2012; 39: 6208-6236.

9. Timmerman RD, Hu C, Michalski J et al. Long-term results of RTOG 0236: A phase II trial of stereotactic body radiation therapy (SBRT) in the treatment of patients with medically inoperable stage I non-small cell lung cancer. Int J Radiat Oncol Biol Phys 2014; 90: S30.

10. Davis JN, Medbery C, Sharma S et al. Stereotactic body radiotherapy for centrally located early-stage non-small cell lung cancer or lung metastases from the RSSearch ${ }^{\circledR}$ patient registry. Radiat Oncol 2015; 10: 113.

11. Lischalk JW, Woo SM, Kataria S et al. Long-term outcomes of stereotactic body radiation therapy (SBRT) with fiducial tracking for inoperable stage I non-small cell lung cancer (NSCLC). J Radiat Oncol 2016; 5: 379-387.

12. Rusthoven KE, Kavanagh BD, Cardenes H et al. Multi-institutional phase I/II trial of stereotactic body radiation therapy for lung metastases. J Clin Oncol 2009; 27: 1572-1578.

13. Timmerman R, McGarry R, Yiannoutsos $C$ et al. Excessive toxicity when treating central tumors in a phase II study of stereotactic body radiation therapy for medically inoperable early-stage lung cancer. J Clin Oncol 2006; 24: 4833-4839.

14. Chang JY, Bezjak A, Mornex F. Stereotactic ablative radiotherapy for centrally located early stage non-small-cell lung cancer: what we have learned. J Thorac Oncol 2015; 10: 577-585.

15. Videtic GM, Paulus R, Singh AK et al. Long-term follow-up on NRG Oncology RTOG 0915 (NCCTG N0927): A randomized phase 2 study comparing 2 stereotactic body radiation therapy schedules for medically inoperable patients with stage I peripheral non-small cell lung cancer. Int J Radiat Oncol Biol Phys 2017; 99: S15-S16.

16. Milickovic N, Tselis N, Karagiannis E et al. Iridium-Knife: Another knife in radiation oncology. Brachytherapy 2017; 16 884-892.
17. Videtic $G$, Singh A, Olivier $K$ et al. A randomized phase II study comparing 2 stereotactic body radiation therapy (SBRT) schedules for medically inoperable patients with stage I peripheral non-small cell lung cancer. RTOG Protocol 0915. 2009.

18. Liu $\mathrm{HH}$, Balter $\mathrm{P}$, Tutt $\mathrm{T}$ et al. Assessing respiration-induced tumor motion and internal target volume using four-dimensional computed tomography for radiotherapy of lung cancer. Int J Radiat Oncol Biol Phys 2017; 68: 531-540.

19. Tran A, Zhang J, Woods K et al. Treatment planning comparison of IMPT, VMAT and $4 \Pi$ radiotherapy for prostate cases. Radiat Oncol 2017; 12: 10.

20. Dong P, Lee P, Ruan D et al. 4ח noncoplanar stereotactic body radiation therapy for centrally located or larger lung tumors. Int J Radiat Oncol Biol Phys 2013; 86: 407-413.

21. Caillet V, Keall PJ, Colvill E et al. MLC tracking for lung SABR reduces planning target volumes and dose to organs at risk. Radiother Oncol 2017; 124: 18-24.

22. Stieb S, Lang S, Linsenmeier C et al. Safety of high-dose-rate stereotactic body radiotherapy. Radiat Oncol 2015; 10: 27.

23. Navarria P, Ascolese AM, Mancosu P et al. Volumetric modulated arc therapy with flattening filter free (FFF) beams for stereotactic body radiation therapy (SBRT) in patients with medically inoperable early stage non-small cell lung cancer (NSCLC). Radiother Oncol 2013; 107: 414-418.

24. Booth JT, Caillet V, Hardcastle $\mathrm{N}$ et al. The first patient treatment of electromagnetic-guided real time adaptive radiotherapy using MLC tracking for lung SABR. Radiother Oncol 2016; 121: 19-25.

25. Ravkilde T, Keall PJ, Højbjerre K et al. Geometric accuracy of dynamic MLC tracking with an implantable wired electromagnetic transponder. Acta Oncol 2011; 50: 944-951.

26. Chan M, Wong M, Leung R et al. Optimizing the prescription isodose level in stereotactic volumetric-modulated arc radiotherapy of lung lesions as a potential for dose de-escalation. Radiat Oncol 2018; 13: 24.

27. Guckenberger M, Klement RJ, Allgäuer M et al. Local tumor control probability modeling of primary and secondary lung tumors in stereotactic body radiotherapy. Radiother Oncol 2016; 118: 485-491.

28. Paddick I, Lippitz B. A simple dose gradient measurement tool to complement the conformity index. J Neurosurg 2006; 105 (Suppl): 194-201.

29. Bhagat N, Fidelman N, Durack JC et al. Complications associated with the percutaneous insertion of fiducial markers in the thorax. Cardiovasc Intervent Radiol 2010; 33: 1186-1191.

30. Kothary N, Heit JJ, Louie JD et al. Safety and efficacy of percutaneous fiducial marker implantation for image-guided radiation therapy. J Vasc Interv Radiol 2009; 20: 235-239.

31. Guckenberger M, Andratschke N, Alheit H et al. Definition of stereotactic body radiotherapy: principles and practice for the treatment of stage I non-small cell lung cancer. Strahlenther Onkol 2014; 190: 26-33.

32. Shibamoto Y, Miyakawa A, Otsuka S, Iwata H. Radiobiology of hypofractionated stereotactic radiotherapy: what are the optimal fractionation schedules? J Radiat Res 2016; 57: i76-i82. 